\title{
The Impact of a Hospitalist Model in Perioperative Care of Elective Joint Replacement Patients: a 17 years-experience Report
}

\author{
Elena Azzolini \\ Istituto Clinico Humanitas \\ Giuseppe Strangio \\ Istituto Clinico Humanitas \\ Salvatore Badalamenti \\ Istituto Clinico Humanitas \\ Michele Lagioia \\ Istituto Clinico Humanitas
}

Matteo Carlo Ferrari ( $\square$ matteo_carlo.ferrari@humanitas.it)

Istituto Clinico Humanitas https://orcid.org/0000-0001-8487-6357

Research article

Keywords: LOS, COPD, obesity, diabetes, cardiovascular diseases, chronic obstructive

Posted Date: July 20th, 2020

DOI: https://doi.org/10.21203/rs.3.rs-32160/v1

License: (9) This work is licensed under a Creative Commons Attribution 4.0 International License.

Read Full License 


\section{Abstract \\ Background}

Hospitalists are physicians whose primary professional focus is the general medical care of hospitalized patients. The aim of this report is to describe a 17-year experience of a hospitalist model in peri-operative co-management in more than 25,000 elective joint replacement patients.

\section{Methods}

A retrospective observational study about surgical co-management with hospitalists in a tertiary teaching referral hospital in Milan in relation to hospital lengths of stay (LOS), 30-days readmission rate, incidence of prosthetic joint infections (PJI) and patient related co-morbidities, number of medico-legal disputes and related costs, and medical customer satisfaction.

\section{Results}

Over a 17-year period LOS has been reduced from 10 to 5 days; readmission rates for total hip arthroplasty are $1.7 \%$ vs $3.7 \%$ (Italy) at 30 days; total infection rates for total knee arthroplasty are recorded at $0.8 \%$ vs $1.4 \%$ (Italy); obesity, diabetes, cardiovascular diseases and chronic obstructive pulmonary disease (COPD) and/or smoking result as the main co-morbidity in PJl; there have been only 113 medico-legal disputes out of approximately 19,000 surgeries and we report $95 \%$ overall medical customer satisfaction.

\section{Conclusions}

This is the first paper outside the US to highlight the impact of the hospitalists on patient satisfaction and the translation of the obtained benefits into savings. The evolution of the hospitalist model in surgical settings shows promising results in terms of patients' safety and cut of health-related direct and indirect costs.

\section{Background}

Surgical co-management with hospitalists is a 20 -year reality in the US. In 1999, Freese ${ }^{1}$ described the first "hospitalist" system, developed at Park Nicollet Clinic in 1993 (Minneapolis, Minnesota) where internists and family practitioners were given the opportunity to merge in a totally office based multispecialty group with the objective to reach a good patient acceptance along with increased physician satisfaction. Additionally, Wacther and Goldman ${ }^{2}$ in 1996 described the importance of efficient care by managed-care organizations for a proper use of resources for inpatients. They established a new 
breed of physicians called "hospitalists" - specialists in inpatient medicine - responsible for managing the care of hospitalized patients in the same way that primary care physicians are responsible for managing the care of outpatients. The National Association of Inpatient Physicians (NAIP), the society representing hospitalists, proposed a definition in 1999: "Hospitalists are physicians whose primary professional focus is the general medical care of hospitalized patients. Their activities include patient care, teaching, research, and leadership related to hospital care." The hospitalists' involvement in the care of different kind of surgical patients during the preoperative and postoperative phases allows the surgeon to be in the operating room all day ensuring at the same time appropriate care to the patient on the ward. A milestone in describing the hospitalist figure with his or her roles and potential activities is again thanks to Wachter ${ }^{3}$ who demonstrated a stunning approximate saving of $\$ 2.2$ billion per year due to hospitalist in US. In Literature many papers are focused on emergency patients admitted for hip fracture with controversial

conclusions. The first published paper that focused on elective hip and knee arthroplasty dates back to 2004 by Huddleston and colleagues ${ }^{4}$. The first review of outcomes and quality measures comparing hospitalists with non-hospitalists cared patients is attributed to Peterson ${ }^{5}$ in 2009. More recently, in 2011 Hinami et al. ${ }^{6}$ described the Hospitalist model in patient services of neurosurgery, urology, and orthopaedic, transplant, and vascular surgeries. In 2016 Duplantier et al. ${ }^{7}$ compared postoperative medical co-management of total hip and knee arthroplasty patients using a hospitalist and nonhospitalist model although controversially with no significant outcome differences. A proof of important cost savings related to this co-management model in orthopaedic and neurosurgical patients is due to the work of Rohatgi and co-workers ${ }^{8}$ in 2016 . These data are confirmed in 2018 by Fitzgerald et al. ${ }^{9}$. In 2019 Salim and colleagues ${ }^{10}$ published a very complete and exhaustive systematic review and metaanalysis of 61 studies comparing many health-assistance related quality indexes in the era before and after the application of the hospitalist model in different medical realities. Finally, we describe our 17 years of experience in the peri-operative co-management together with Anaesthesiologists and Orthopaedics of more than 25,000 elective joint replacement patients confirming the high quality impact of Hospitalist approach.

\section{Methods}

A retrospective observational study was carried out in relation to the Italian national register of highvolume orthopaedic surgery hospitals and considered the following criteria: hospital lengths of stay (LOS) in the last 5 years, 30-days readmission rate for any medical reason (last 5 years), incidence of prosthetic joint infections (PJI) and patients related co-morbidities in PJI in the last 10 years, number of medico-legal disputes and related costs, and medical customer satisfaction in the last 10 years.

\section{Results}


LOS was reduced from 10 days between 2003 and 2012, to 7 days between 2013 and 2016, to 5 days after 2017 with the implementation of the rapid recovery together with our Anaesthesiologist team and Rehabilitation Department (Table 1).

Our 30-days readmission rate (2015-2019, approximately 14,000 patients in our Hospital) for total hip arthroplasty (THA) is recorded as $1.7 \%$ vs high volume hospitals in Italy ( $>300$ arthroplasties/year) $3.2 \%$ vs Italy national mean $3.7 \%$. Considering total knee arthroplasty (TKA) we recorded $0.8 \%$ vs high volume hospitals in Italy $1.3 \%$ vs Italy national mean $1.4 \%$ (Table 2 ).

Concerning infections in our hospital, the total infection rate results as approximately $2 \%$ and the PJI accounts for $0.8 \%$ of total arthroplasties in the index period. Further to this, in 2009 a $0.69 \%$ of PJI was reported but we observed a rise to $1.2 \%$ in 2010 . As a result, our antibiotic prophylaxis approach changed (duration, timing, dosage) and starting from 2011 we obtained a statistically significant reduction $(P=0.01)$ to $0.39 \%$, and maintained this figure in $2012(0.39 \%)$, in 2013 the figure was $(0.51 \%)$, stabilizing to less than $1 \%$ with a mean of $0.8 \%$ over the past 10 years.

Considering patients co-morbidities in PJI, we observed the following: obesity $46.2 \%$, diabetes $34.6 \%$, cardiovascular diseases $34.6 \%$, COPD/smoking $26.9 \%$, renal insufficiency $19.2 \%$, autoimmune diseases $19.2 \%$, previous malignancy $15.4 \%$, liver diseases $11.5 \%$ (Table 3 ).

As for the number and type of medical consultations they resulted as episodic, mainly due to cardiology and infectious diseases (an infectious disease specialist joined our team 2 years ago).

Finally, the number of medico-legal disputes and related costs are recorded at 113 out of approximately 19,000 surgeries over the last 10 years $(0.6 \%)$.

The overall medical customer satisfaction of $95 \%$.

\section{Discussion}

In Minneapolis in 1993, Freese $^{1}$ described the first experience in the use of physician specialists in inpatient medicine.

In 1996, Wacther and Goldman² underlined the importance of hospitalists in efficient care for a proper use of resources for inpatients: as hospital stays become shorter and inpatient care becomes more intensive, a greater premium will be placed on the skills, experience, and availability of physicians caring for inpatients with the ability to respond quickly to changes in patient's conditions. In an academic setting, hospitalists provide a premium on clinical quality improvement, in the development of practice guidelines, and outcome research.

Wachter ${ }^{3}$ in 2002 reviewed the Literature on the evolution of the hospitalist model in the US underlying the significant decrease in hospital LOS and costs savings, accounting at that time (calculated on the US 4500 hospitalist base) for a stunning $\$ 2.2$ billion per year. In fact, with more than 30,000 hospitalist staff 
approximately $70 \%$ of US hospitals that number should be multiplied by 6 ( $\$ 13$ billion). Moreover, Wachter described the core and potential additional activities for hospitalists.

The first published paper that focused on surgical co-management with hospitalist in elective hip and knee arthroplasty dates back to 2004 . Huddleston and colleagues ${ }^{4}$ demonstrated that more patients in the hospitalist group were discharged from the hospital with no complications (61.6\% vs. $49.8 \%)$, fewer minor complications were observed among hospitalist patients (30.2\% vs. $44.3 \%)$, and mean length of stay for patients in the hospitalist model of care was shorter (5.1 days vs. 5.6 days). Although total costs did not differ between groups, orthopaedic surgeons and nurses preferred the hospitalist model.

The first review of outcomes and quality measures comparing hospitalists with non-hospitalists cared patients is thanks to Peterson ${ }^{5}$ in 2009 including orthopaedic surgery, pneumonia and heart failure. The author showed that the inpatient care by hospitalists leads to decreased hospital cost and LOS. Hospitalist care was also reported to improve several measures of care: orthopaedic surgery patients had a shorter time related to surgery, consultation, and hospital LOS. Hospitalists have more practice and experience tending to inpatient medical problems than the so-called disease-specific physician experience.

In 2011 Hinami et al ${ }^{6}$ studied inpatient services of neurosurgery, urology, and orthopaedic, transplant, and vascular surgeries. Medical co-management was associated with fewer inhospital deaths (odds ratio $0.23)$, shorter stays $(-2.6$ days, $P<.01)$ without significant differences in total cost. Their analysis suggests that the widespread adoption of postoperative co-management may find benefits in the role of hospitalists as facilitators of rescue. Hospitalists may play an important supportive role in facilitating care for an increasingly elderly and comorbid surgical population, because of their exclusive focus on surgical patients, their orientation towards improving postsurgical care quality, and the higher intensity of involvement in their patients' care.

Rohatgi and co-workers ${ }^{8}$ firstly demonstrated in 2016 a significant savings estimate of $\$ 2,600-4,300$ per patient in a cohort of orthopaedic and neurosurgical patients. Surgical co-management with hospitalists was associated with a significant differential decrease in the proportion of patients with at least one medical complication after surgery $(P=0.008)$, the reduction of LOS $(P<0.001)$ and of 30-days readmission rate for medical reasons $(P<0.001)$, and the reduction of medical consultations $(P<0.001)$. The overall patient satisfaction was elevated $(88.3 \%)$.

On the contrary, Duplantier et al. ${ }^{7}$ (2016) retrospectively compared postoperative medical comanagement of total hip and knee arthroplasty patients using a hospitalist $(\mathrm{H}, 1,656$ patients $)$ and nonhospitalist model (NH, 1,319 patients), and showed decreased LOS $(P<0.001)$ in $\mathrm{H}$ group, but a total rise in direct costs due to a greater number of tests ordered $(P<0.001)$ resulting in more new diagnosis $(P<$ $0.001)$ and with higher costs of hospitalization $(P=0.002)$; no differences were seen in readmission rates. 
In 2018, Fitzgerald et al. ${ }^{9}$ studied 1,100 patients before and after Hospitalist-Orthopaedic Comanagement submitted to TKR or THA showing statistically significant improvements in multiple performance and quality metrics: reduction of mean LOS (from 3.36 to 3.17 days), increase in percentage of patients discharged at home (from 53.9-59\% with reduction of unnecessary resource utilization over the episode of care), decrease in medical complications rate $(P<0.05)$, and 30-days readmission rate $(P<$ 0.05 ), improved rescue and understanding about the needs of surgical patients, increased collaboration between surgeon and hospitalist and anaesthesiologist, avoiding unnecessary testing resulting in better costs control.

Very recently, in 2019 Salim et al. ${ }^{10}$ conducted a systematic review and meta-analysis in the impact of hospitalists on the efficiency of inpatient care and patient satisfaction, based on 61 studies. Their analysis showed that hospitalist care is associated with decreased LOS and increased patient satisfaction compared to non-hospitalist model. Hospitalists provide more efficient, less costly inpatient care with equal or higher quality, reducing LOS, readmissions and in-hospital mortality. They stated that there is compelling evidence that hospitalists promote clinical care development and integration: particularly, they supported the development of patient safety guidelines and became more efficient in teaching. As inpatient leaders, hospitalists collaborate well with emergency physicians in discharging those patients, which could well be managed as outpatient. Hospitalists are also used to managing complex patients themselves both in acute postoperative complications and in chronic comorbidities, minimizing use of subspecialists; moreover, hospitalists are well aligned with all the figures involved in health care system focused on decreasing length of stay, improving resource utilization and decreasing readmissions, leading to cost savings.

Consequently, we describe our 17 years of experience in more than 25,000 elective prosthetic joint replacements. In Lombardy (Milan Region of Italy), orthopaedic patients account for about $12 \%$ of total medical claims for damages with a mean of reimbursement of 60,000 euro (SD 99,000 euro). More in detail, $54 \%$ are related to surgical errors, $10 \%$ to diagnostic mistakes and $9 \%$ to infections and therapy errors each. In our hospital the total infection rate is about $2 \%$ and the PJI accounts for $0.8 \%$ of total arthroplasties in the index period. Additionally, in 2009 we had $0.69 \%$ of PJI, but we observed a rise of $1.2 \%$ in 2010 and our antibiotic prophylaxis approach (duration, timing, dosage) was changed. From 2011 we obtained a statistically significant reduction $(P=0.01)$ to $0.39 \%$, and maintained $0.39 \%$ in 2012 and $0.51 \%$ in 2013 , stabilizing to less than $1 \%$ with a mean of $0.8 \%$ in the last 10 years. This excellent

result is probably also due to the presence in our hospital of two hospitalists focused and expert in PJI prevention and treatment. The overall medical $\mathrm{cl}$

aims for damages is approximately $0.6 \%$, far below our standard average of $12 \%$, accounting for about 125 million euro saved in the last 10 years. The gradual implementation of the rapid recovery program together with anaesthesiologists, orthopaedics and physiotherapists allows us to obtain a progressive reduction of LOS from 10 to 5 days in the index period with a mean cost per day of hospitalization of 400 euro per patient, accounting for a total of about 17.5 million euro saved between 2013 and 2019 
(Table 1). The 30-day readmission rate for any medical/surgical reasons in THA is $1.7 \%$ (much less than the $3.2 \%$ of high volume hospitals in Italy and $3.7 \%$ of the Italian national mean). We obtain the same very good

results for TKA: $0.8 \%$ vs $1.3 \%$ of high volume hospitals in Italy and $1.4 \%$ of Italian national mean (Table 2). External medical consultations are episodic, mainly due to cardiology and infectious disease specialists (none in the last 2 years as an ID specialist joined our team). The overall patient satisfaction is very high and rates at $95 \%$. These very good

results are related to a strict relevance to evidence-based and internal protocols in patient management starting from the pre-hospitalization through the hospitalization period (ward, surgical theatre, rehabilitation) until the discharge at home. In this patient care management continuum, the hospitalist figure plays a leading role due to the 360 degrees medical outlook of an internist. In our reality we are able to confirm that orthopaedic surgeons, anaesthesiologists and nurses appreciate the hospitalist model for different reasons: firstly, both orthopaedics and anaesthesiologists can be more focused on the operating theatre relying on the presence of an experienced internist to take care of their ward patients; secondly nurses can count on the permanent presence of a referral doctor. This study has some limitations: first, it is a retrospective study; second, our data has become more accurate in the last 5 to 10 years due to the implementation of computerized data storage. Our study also has some strengths: to our knowledge, it is the paper with the biggest number of patients followed by hospitalists over a long period of time (17 years) demonstrating the great importance of such a new specialization for internists. Moreover, this is the first paper considering the cost savings related to a significant decrease in medical claims thanks to the hospitalist figure.

\section{Declarations}

\section{- Ethics approval and consent to participate:}

our Ethical Committee was noticed about the study and all the patients gave their consent to the study.

\section{- Consent for publication:}

not applicable

\section{- Availability of data and materials:}

all data reported in the present study are available in our database

\section{Competing interests:}

no conflicts exist for any of the authors

\section{- Funding:}


not applicable

\section{Authors' contributions:}

All authors contributed to the study conception and design. Material preparation, data collection and analysis were performed by MCF and EA. The first draft of the manuscript was written by MCF and all authors commented on previous versions of the manuscript. All authors read and approved the final manuscript.

\section{- Acknowledgements:}

not applicable

\section{References}

1. Freese RB. The Park Nicollet experience in establishing a hospitalist system. Ann Intern Med. 1999 Feb;16(4 Pt 2):350-4.. ;130.

2. Wachter RM, Goldman L. The emerging role of "hospitalists" in the American health care system. N Engl J Med. 1996 Aug;15(7):514-7.., ;35.

3. Wachter RM. The evolution of the hospitalist model in the United States. Med Clin N Am. 2002 Jul;86(4):687-706.

4. Huddleston JM, Long KH, Naessens JM, Vanness D, Larson D, Trousdale R, Plevak M, Cabanela M, Ilstrup D, Wachter RM. Hospitalist-Orthopedic Team Trial Investigators. Medical and surgical comanagement after elective hip and knee arthroplasty: a randomized, controlled trial. Ann Intern Med. 2004 Jul;6(1):28-38. ; ;41.

5. Peterson MC. A systematic review of outcomes and quality measures in adult patients cared for by hospitalists vs nonhospitalists. Mayo Clin Proc. 2009 Mar,84(3):248 - 54.

6. Hinami K, Feinglass J, Ferranti DE, Williams MV. Potential role of comanagement in "rescue" of surgical patients. Am J Manag Care. 2011 Sep;1(9):e333-9.. ;17.

7. Duplantier NL, Briski DC, Luce LT, Meyer MS, Ochsner JL, Chimento GF. The Effects of a Hospitalist Comanagement Model for Joint Arthroplasty Patients in a Teaching Facility. J Arthroplasty. 2016 Mar,31(3):567 - 72.

8. Rohatgi N, Loftus P, Grujic O, Cullen M, Hopkins J, Ahuja N. Surgical Comanagement by Hospitalists Improves Patient Outcomes: A Propensity Score Analysis. Ann Surg. 2016 Aug;264(2):275 - 82.

9. Fitzgerald SJ, Palmer TC, Kraay MJ. Improved Perioperative Care of Elective Joint Replacement Patients: The Impact of an Orthopedic Perioperative Hospitalist. J Arthroplasty. 2018 Aug;33(8):2387-91.

10. Salim SA, Elmaraezy A, Pamarthy A, Thongprayoon C, Cheungpasitporn W, Palabindala V. Impact of hospitalists on the efficiency of inpatient care and patient satisfaction: a systematic review and meta-analysis. J Community Hosp Intern Med Perspect. 2019 Apr 12;9(2):121-134. 


\section{Tables}

Table 1

Length of stay in hospital (LOS)

\begin{tabular}{|lll|}
\hline $2003-2012$ & $2013-2016$ & $2017-2019$ \\
\hline 9600 patients & 8300 patients & 8900 patients \\
\hline 10 days & 7 days & 5 days \\
\hline
\end{tabular}

\section{Table 2}

30-days readmission rate 2015-2019 (14000 patients)

Istituto Clinico Humanitas (ICH), High Volume (>300/year) Arthroplasty Hospitals (HVAH), Italy Overall Mean (IOM), Total Hip Arthroplasty (THA), Total Knee Arthroplasty (TKA)

\begin{tabular}{|llll|}
\hline & ICH & HVAN & IOM \\
\hline THA & $1.7 \%$ & $3.2 \%$ & $3.7 \%$ \\
\hline TKA & $0.8 \%$ & $1.3 \%$ & $1.4 \%$ \\
\hline
\end{tabular}

\section{Table 3}

Prosthetic joint infections (PJI) risk factors

\begin{tabular}{|ll|}
\hline NO RISK FACTORS & $11.5 \%$ \\
\hline OBESITY & $46.2 \%$ \\
\hline DIABETES & $34.6 \%$ \\
\hline CARDIOVASCULAR DISEASES & $34.6 \%$ \\
\hline COPD/SMOKING & $26.9 \%$ \\
\hline RENAL INSUFFICIENCY & $19.2 \%$ \\
\hline AUTOIMMUNE DISEASES & $19.2 \%$ \\
\hline PREVIOUS MALIGNANCIES & $15.4 \%$ \\
\hline LIVER CHRONIC DISEASES & $11.5 \%$ \\
\hline
\end{tabular}

